\title{
Les obstacles à la réalisation du cycle vital des poissons
}

\author{
Obstacles facing the completion \\ of vital cycles of fish
}

G. Leynaud

Chef du Département
Ressources en eau
F. Trocheris

Ingénieurs à la Division

Qualité des eaux, pêche et pisciculture

\section{CEMAGREF}

\begin{abstract}
Les pressions physiques et chimiques d'origine naturelle ou artificielles s'exerçant sur les cours d'eau
limitent le développement d'un nombre important d'espèces particulièrement parmi les migrateurs amphibiotiques.

Les différents types d'atteinte au milieu aquatique entraînant la raréfaction de ces espèces maintenant protégées au titre de la loi sur la protection de la nature sont illustrées par quelques exemples.

Le déroulement du cycle vital des diverses espèces de poissons comprend plusieurs phases : reproduction et développement embryonnaire, développement juvénile, développement adulte et plusieurs fonctions (reproduction, alimentation, repos) généralement situées en des sites différenciés.

L'accomplissement du cycle complet est tributaire de l'adéquation de l'environnement aux besoins des espèces au cours de ces différentes phases (habitat et qualité des eaux) et des possibilités de circulation entre les sites correspondantes.
\end{abstract}

Natural and artificial physical and chemical pressures affecting rivers thus restrict the growth of a large number of species especially amongst migrating amphibiotic specifies.

The various species affected in the aquatic environment resulting in the rarefaction of those species which are currently protected under the Law for Preserving Natural Environment are illustrated by some examples.

The course of the vital cycle of the various species of fish includes several phases; reproduction and embryonic evolution, juvenile development, adult development and several functions (reproduction, feeding, sleep) generally situated in two different locations.

The completion of a complete cycle depends on a perfectly suitable environment responding to the needs of the species throughout these various phases (habitat and quality of water) and possibilities to circulate between the corresponding locations. 
Les exigences spatiales des différentes espèces de poissons représentées dans la faune de France sont très variables. $\mathrm{Si}$ certaines espèces euryèces s'adaptent à des conditions d'habitat variées, d'autres comme la truite, l'ombre commun,... exigent un habitat nettement différencié suivant le stade de leur développement et la fonction qu'elles assument : il est ainsi possible de caractériser avec précision des zones de reproduction, des zones de développement des juvéniles, des zones de repos ou d'abri et des zones de nutrition.

Le déroulement du cycle vital des espèces dites « sténotopes " nécessite d'une part l'intégrité de leurs différents habitats et d'autre part la possibilité de circuler librement entre les sites correspondants.

Ce deuxième point est particulièrement manifeste dans le cas des poissons migrateurs amphibiotiques, dont les zones de croissance et de reproduction peuvent se situer à plusieurs milliers de kilomètres de distance.

Il n'est donc pas étonnant que ces espèces aient été les premières à souffrir de l'utilisation croissante à des fins industrielles des cours d'eau, multipliant le nombre et la nature des obstacles à leur migration anadrome ou catadrome.

\section{Les obstacles physiques à la réalisation du cycle vital des poissons}

Les obstacles naturels (cascades de grande hauteur) peuvent sur certains cours d'eau limiter l'aire de distribution des migrateurs. Pour un certain nombre d'entre eux, des aménagements artificiels ont été réalisés pour permettre le passage des poissons notamment sur le continent nord-américain (cas de la rivière Fraser).

En France, les obstacles les plus importants sont constitués par les divers ouvrages et aménagements hydrauliques liés à l'activité humaine.

\subsection{Les obstacles à la remontée des migrateurs}

Les difficultés les plus évidentes qu'éprouve un poisson à remonter un cours d'eau ont pour origine les obstacles physiques constitués par les barrages.

La notion d'obstacle n'est pas seulement liée à la hauteur du barrage. Elle dépend :

- des conditions hydrodynamiques sur l'ouvrage (vitesses, tirants d'eau);

- du profil de l'ouvrage. Des profils concaves ou présentant des ruptures brutales de pente (rehausses) peuvent se révéler pratiquement infranchissables;

- des populations des espèces migratrices considérées, de leur capacité de nage ou de saut, de leur état physiologique;

- de la température de l'eau : certains ouvrages, franchissables à certaines périodes de l'année, deviennent des obstacles dès que les eaux sont froides et que les capacités de nage des poissons sont réduites.

Pour ces diverses raisons, la plupart des auteurs considèrent que des blocages peuvent intervenir dès que la chute dépasse 0,50 mètre (Bell, 1973, Beach, 1984).
Parmi les obstacles à la migration, il est possible de distinguer :

- des barrages de retenue, destinés à créer des réserves saisonnières ou d'éclusées (barrage de Poutès sur l'Allier); - des barrages de prise, ne comportant pas de réserve mais permettant la dérivation du débit vers des turbines. Ces barrages peuvent être fixes, semi-mobiles ou mobiles (clapets);

- des barrages-usines de dénivellation, destinés à créer leur propre chute. Certains ouvrages servent à la fois à la production d'énergie électrique et à la navigation, d'autres étant uniquement destinés à rehausser le niveau des eaux pour la navigation (Aulne canalisée, Blavet);

- des barrages édifiés sur de grands cours d'eau dans le but de créer des plans d'eau à utilisation touristique (Blois sur la Loire, Vichy Pont du Château sur l'Allier, Beaulieu sur la Dordogne);

- des chaussées d'anciens moulins, en particulier en Bretagne et en Normandie. Dans la mesure où ils ne sont plus utilisés la meilleure des solutions pour supprimer ces obstacles est de les effacer (cas de nombreux seuils dans les Côtes du Nord);

- des seuils fixes destinés à maintenir le niveau de la nappe phréatique (seuils en aval du barrage de Malause sur la Garonne) ainsi que ceux édifiés dans le but dé fixer le lit mineur des cours d'eau destabilisés suite à des extractions intensives de matériaux alluvionnaires (Gave de Pau, Adour, Gardon, Durance...);

- des radiers de ponts (pont de Règemortes, radier du barrage de Vichy sur l'Allier) créant obstacle à la suite de l'enfoncement du lit du cours d'eau consécutif à des extractions de granulats;

- le busage sans précautions (pentes trop fortes, tirants d'eau insuffisants en étiage, chute à l'aval de la buse) de cours d'eau pour le passage d'autoroutes.

- les seuils de prise d'eau des centrales nucléaires (St Laurent des Eaux, Dampierre en Burly et Belleville sur la Loire);

- les clapets à marée.

De tels obstacles ne peuvent être franchis que s'ils sont correctement équipés de passes à poissons, mais en aucun cas ces dispositifs ne permettent de revenir à la situation antérieure : ce n'est la plupart du temps qu'une partie du stock présent qui parvient à trouver l'entrée des passes à poissons et bien souvent le franchissement d'un barrage s'accompagne d'un retard important à la remontée. Toutes les expériences de télémétrie montrent que les retards à la migration peuvent varier de quelques heures dans les meilleurs des cas à plusieurs semaines (Larinier, Travade, 1982; Guéneau, 1984).

Le cumul des obstacles peut engendrer d'une part la réduction du stock de géniteurs qui parvient jusqu'aux frayères et d'autre part une arrivée tardive sur ces lieux, ce qui peut compromettre la réussite de la reproduction : ceci s'applique particulièrement à une espèce comme l'alose, qui ne dispose que d'un temps très court entre le moment où elle pénètre en eau douce et celui où elle se reproduit.

Les barrages peuvent en outre occasionner d'autres nuisances que le simple obstacle physique qu'ils créent.

Tout d'abord la mise en eau des retenues peut provoquer la disparition de zones de frayères. 
Par ailleurs, lorsque les barrages créent une dérivation sur le cours d'eau, la partie court-circuitée ne connait plus qu'un débit limité qui peut poser problème au passage des migrateurs, en raison de la faible hauteur d'eau présente au niveau des zones de radiers.

De tels cas ont été cités en Bretagne (anonyme, 1968) où des piscicultures absorbaient la presque totalité du débit de certaines rivières, laissant pratiquement à sec les secteurs court-circuités et empêchant ainsi la remontée des géniteurs saumons.

Le mode de gestion des retenues peut en outre influer sur la qualité biologique des secteurs situés en aval : Cuinat (1980) signale la mise à sec périodique de près de la moitié des zones courantes jusqu'à une trentaine de kilomètres à l'aval de l'usine EDF de Poutès Monistrol, après chaque période de turbinage de pointe.

Enfin certains barrages, dits de fond d'estuaires, ont pour fonction de limiter la remontée d'eau salée dans les terres et constituent une séparation nette entre deux milieux de salinités très différentes. Ces barrages posent d'abord problème pour le passage des migrateurs: les vannes ou clapets à marée ne s'ouvrent bien souvent que lorsque la charge hydraulique amont est nettement supérieure à celle d'aval, ce qui engendre des vitesses trop importantes au droit des ouvertures.

Par ailleurs, les poissons sont obligés de passer brutalement du milieu marin à l'eau douce; bien que ce phénomène soit très peu étudié, il est probable que, dans des situations naturelles, bon nombre de migrateurs aient besoin de s'acclimater à une telle transition (Dodson et coll., 1972).

Il faut d'ailleurs souligner à ce propos la grande sensibilité, vis-à-vis de la qualité de l'eau, des migrateurs en période de remontée, étant donné les besoins physiologiques correspondant au changement de milieu et à la maturation sexuelle.

Cette sensibilité est particulièrement marquée lorsqu'il s'agit des alevins comme dans le cas de l'anguille. Les civelles au cours de la montaison subissent d'importantes transformations physiologiques et morphologiques coordonnées avec l'évolution normale des salinités. Dans leur étude des effets du barrage d'Arzal, Elie et Rigaud (1984) ont mis en évidence le rôle d'obstacle du barrage et de ceux situés plus en amont ainsi que les anomalies de développement dues à la suppression de la diminution progressive de salinité.

La biologie et le comportement de ces organismes peuvent nécessiter l'aménagement de dispositifs de franchissement adaptés.

\subsection{Les obstacles physiques à la descente des migra- teurs}

L'avalaison a été particulièrement étudiée chez les jeunes saumons.

Les principaux problèmes que rencontrent au cours de leur descente les juvéniles sont :

- le transit dans les turbines des centrales hydroélectriques;

- l'augmentation de la durée de migration du fait de la traversée des retenues et de la recherche d'exutoires;

- la sursaturation de l'eau en azote en aval des déversoirs des barrages;

- l'entraînement dans les prises d'eau.

En premier lieu le franchissement des barrages par les juvéniles pose de sérieuses difficultés.

Le passage est possible par deux voies : les turbines ou les déversoirs.

Dans le cas du transit par les turbines, les causes de mortalité sont les contacts mécaniques avec les équipements fixes ou mobiles (distributeurs, aubes ou pales...), les actions de cisaillement dues au passage dans des zones à fort gradient de vitesse, les décélérations ou accélérations brutales, les dommages induits par les conditions de pression sub-atmosphérique - et tout particulièrement les phénomènes de cavitation -

Les taux de mortalité peuvent varier de quelques pour cent à plus de 75 pour cent.

Des taux de 5 à 10 pourcent paraissent inévitables. Certaines espèces, en particulier les aloses, semblent être beaucoup plus fragiles que les salmonidés sur lesquels ont porté la plupart des expérimentations.

Il est intéressant en outre de noter que le stress subi par les poissons paraît " discriminant " : ceux qui survivent au passage au travers des turbines ne seraient pas physiologiquement affectés par cette opération.

Le passage par les déversoirs occasionne des taux de mortalité du même ordre de grandeur que le transit à travers les turbines, si l'on se réfère aux résultats rapportés par Bell \& Delacy (1972, in Ruggles, 1980). Si les juvéniles de saumons sont capables de résister à des changements considérables de pression, ils peuvent néanmoins subir plusieurs types de dommages lorsqu'ils transitent par un déversoir : choc contre la base du déservoir, abrasion sur la paroi rugueuse du déversoir, cisaillement par des veines d'eau de vitesse très différentes, impact sur la surface de l'eau si le poisson tombe en chute libre...

Les résultats les plus favorables sont obtenus lorsque sur le déversoir et au niveau de la zone de réception est assuré un minimum de turbulence, de remous et de dissipation d'énergie.

Toutefois ces facteurs de mortalité sont loin d'être les seuls occasionnés par les ouvrages de retenue. En effet, le ralentissement de la vitesse du courant en amont de chaque aménagement, et la difficulté de trouver un exutoire, occasionnent des retards importants dans la descente des smolts. Larinier \& Travade (1982) rapportent que sur la côte ouest des Etats-Unis, la durée moyenne de descente d'un juvénile de saumon sur la rivière Salmon est passée de 38 jours à 78 jours après l'équipement de ce cours d'eau.

Ce délai, ainsi que l'affaiblissement dû aux traumatismes, augmente les risques de prédation : c'est ainsi que Mills (1964, in Ruggles, 1980) a estimé la part consommée par les brochets (Esox lucius) à $10 \%$ de la population de smolts dévalant une rivière d'Ecosse équipée de deux retenues hydroélectriques.

Par ailleurs, une arrivée trop tardive en eau de mer peut être très pénalisante pour les jeunes saumons : les branchies de ces poissons présentent une évolution importante de l'activité ATP asique, l'augmentation de cette enzyme leur permettant de mieux se réguler dans un environnement hyperosmotique. Il importe donc que les jeunes saumons parviennent en mer au moment du maximum 
d'activité de l'ATP ase branchial, car ils risquent ensuite de ne pas pouvoir supporter le changement de milieu.

Ces différents phénomènes ont incité les biologistes écossais à ne plus systématiquement imposer l'installation d'écrans devant les aspirateurs des turbines des centrales hydroélectriques : le retard occasionné par la recherche de la passe à poissons qui devient la seule voie de sortie semble être dans de nombreux cas plus dommageable que le transit à travers les turbines.

Autre nuisance occasionnée par les barrages équipant un cours d'eau, la sursaturation de l'eau en azote peut engendrer des mortalités importantes chez les smolts. Bell \& Coll. (1975, in Ruggles, 1980) signalent ainsi que 40 à $95 \%$ des jeunes saumons des rivières Snake et Columbia meurent au cours de leur descente et que la majeure partie des mortalités peut être attribuée à la sursaturation de l'eau en azote dissous.

Cette sursaturation est principalement due à l'entraînement et à la dissolution de l'air dans les couches profondes des bassins de dissipation d'énergie récupérant l'eau transitant par les déversoirs. Un taux de sursaturation de $120 \%$ en azote est susceptible de provoquer des embolies gazeuses chez les salmonidés.

Il faut aussi souligner le fait que, plus encore peut-être que les géniteurs, les jeunes saumons sont extrêmement sensibles à toute altération du milieu, la smoltification les rendant particulièrement fragiles. Lorz \& Mac Pherson (1976, in Ruggles, 1980) ont ainsi montré que des teneurs en cuivre habituellement sans danger pour les poissons ont une action sur l'activité ATP asique et réduisent les chances d'adaptation des smolts à l'eau salée.

Le dernier type d'obstacle à la migration qui doit être cité pour les jeunes saumons est constitué par les prises d'eau. Si le débit de ces dernières est important par rapport au débit du cours d'eau, une proportion notable de smolts peut se trouver entraînée et piégée par la prise d'eau.

Ce phénomène a été particulièrement étudié par Cuinat et ses collaborateurs au niveau des prises d'eau des centrales nucléaires de Chinon et de St. Laurent des Eaux. En 1976, lorsque le débit de la Loire était faible, plus de 3000 smolts ont été piégés dans les chambres d'eau (Cuinat, 1980).

En ce qui concerne l'alose, Albiges et coll. (1984), Boigontier et coll. $(1982,1984)$ ont montré l'importance des contigents d'alosons piégés et détruits au cours de leur avalaison par les dispositifs de filtration de la prise d'eau de la centrale nucléaire du Blayais.

Il faut également citer les entraînements d'alevins par les stations de pompage situées sur les fleuves et destinées à l'irrigation ou d'autres usages. Peu d'études précises ont été réalisées mais les simples observations ont montré que ces prélèvements pouvaient être numériquement importants (ex. sur le Bas-Rhône).

\section{Obstacles dus à la dégradation de la qualité des eaux et des habitats aquatiques}

La pollution des eaux constitue un obstacle à la migration aussi nuisible que les barrages (Roule, 1929).
Il est bien évident que lorsque règnent des conditions létales pour les poissons, toute migration à travers la zone polluée devient impossible. Les concentrations létales sont d'ailleurs abaissées pour les poissons migrant ou s'apprêtant à migrer en raison des bouleversements physiologiques qui accompagnent ce mouvement (Fontaine, 1984). En conditions sublétales, les performances physiques nécessaires au déplacement (nage, saut) sont réduites. Les migrations de saumon ont été interrompues pendant des décennies sur certaines rivières bretonnes par suite de leur pollution organique et chimique.

A des concentrations encore plus faibles, de nombreux toxiques affectent diversement le comportement des poissons (Leynaud et Trocherie, 1980). Ainsi les salmonidés ont une réaction de fuite très nette devant les solutions de sulfate de zinc, l'intensité de la réaction se développant avec l'augmentation des concentrations.

En revanche, les phénols bien que détectés n'induisent pas de réaction marquée de fuite quelle que soit leur concentration.

Avec des toxiques comme le chlore ou les détergents, un comportement paradoxal est observé, les salmonidés manifestant à leur égard une réaction de fuite aux faibles concentrations et une certaine attirance pour les concentrations mortelles (Sprague et Drury, 1968). Des perturbations des mouvements migratoires liées à ces troubles du comportement ont pu être mises en évidence in situ (Fontaine, 1984).

Enfin, certains pesticides (carbamates) inhibent l'activité motrice des poissons (CTGREF, 1977).

La migration reproductrice chez les salmonidés (et probablement chez d'autres migrateurs) correspond à un retour au lieu de naissance (désigné couramment par le terme de "homing "). Ce phénomène a été étudié par de nombreux auteurs qui ont mis en évidence dans le guidage des poissons le rôle extrêmement important des récepteurs chemosensoriels et notamment de l'olfaction: Brannon (1985), Stabel (1984), Saglio (1979, 1983), Nordeng (1977). Fontaine (1984) a montré l'importance du système endocrinien dans le déterminisme de la migration. Or, d'importants travaux scientifiques ont établi l'action perturbatrice de très nombreux polluants sur ces récepteurs sensoriels (détergents, hydrocarbures,...) ou ces systèmes complexes ( $\mathrm{PCB}$, Mirex, dieldrine). Ils ont été synthétisés récemment par Fontaine (1984) qui souligne également les effets possibles d'une altération du régime thermique des eaux. De plus, comme dans le cas des traumatismes subis lors du franchissement des ouvrages, les perturbations induites par la pollution favorisent la prédation sur les migrateurs par les espèces sédentaires plus résistantes.

Les modifications rapides directes (pollution) ou indirectes (destruction ou modification de la végétation,...) des caractéristiques physico-chimiques des zones occupées par les stades juvéniles sont susceptibles de perturber les mouvements migratoires. Ces caractéristiques sont, en effet, "mémorisées" par les migrateurs pendant leur séjour en eau douce et utilisées ensuite pour le guidage vers les lieux de naissance.

Il faut insister également sur l'importance majeure de la conservation des frayères constituées généralement de graviers non colmatés qui sont gravement menacées sur les grands systèmes fluviaux français (Garonne, Loire, Gaves) par les extractions de granulats dans les lits mineurs de 
ces cours d'eau ou par la construction de nouveaux barrages.

La raréfaction des zones propices à la reproduction ou à l'élevage des jeunes augmente d'ailleurs ( $\mathrm{y}$ compris pour les espèces considérées à tort comme totalement sédentaires) les besoins de circulation pour la réalisation du cycle vital complet des poissons; elle peut compromettre le résultat des efforts réalisés par ailleurs en matière de franchissement des ouvrages.

\section{$*$}

Ce rapide et forcément incomplet (faute d'un effort de recherche à la hauteur de la complexité des problèmes) inventaire des obstacles à la réalisation du cycle vital des poissons migrateurs montre cependant la multiplicité des difficultés à surmonter. Si les barrages sont souvent cités à juste titre comme la principale cause de la raréfaction de ces espèces, la totalité de leurs effets indirects est rarement appréhendée.

En outre, toutes les atteintes chimiques et physiques à la qualité des eaux ou à l'habitat aquatique aboutissent à l'établissement de zones difficilement franchissables voire mortelles.

Les ralentissements ou arrêts des mouvements migratoires liés à ces différents types d'obstacles augmentent la capturabilité des poissons par la pêche et rendent plus difficile encore la gestion de stocks réduits en diversité et en effectif total.

Aussi les programmes de restauration des stocks des espèces migratrices devraient-ils comporter un recensement soigneux de l'ensemble des contraintes et nuisances en cause en vue de l'établissement d'un plan de restauration rationnel et cohérent.

\section{Auteurs cités}

Albiges C., Rochard E., Elie P., Boigontier B., 1984. - Etude de surveillance halieutique de l'estuaire de la Gironde 1984. CEMAGREF - ALA, Bordeaux.

ANONYME, 1968. - Etat actuel des rivières à' saumons de Bretagne. PENN AR BED, vol. 6, n 55, p. 373-384.

BEACH M.H., 1984. - Fish pass design. Fisheries Research Technical Report $n^{\circ} 78$. Ministry of Agriculture, Fisheries and Food. 46 p.

BELL M.C., 1973. - Fisheries Handbook of Engineering requirements and biological criteria. Fisheries Engineering Research Programm. Corps of Engineers, North Pacific Division. Portland, Oregan

Boigontier B., Mounie D., 1982. - Etude des macroorganismes entrainés sur les filtres rotatifs de la centrale nucléaire du Blayais (Gironde). DEA Ichtyologie Appliquée. ENSA Toulouse CEMAGREF ALA Bordeaux, 193 p.

Boigontier B., FilipozzI P., TAVerny C., 1985. - Etude de l'efficacité de nouveaux dispositifs de récupération des macroorganismes à la centrale du Blayais (Gironde) (juin 1984. mai 1985). CEMAGREF - ALA Bordeaux/E.D.F. 150 p. + annexes.

BRANNON E.L., 1985. - Les mécanismes d'orientation dans le " homing " des salmonidés. Saumons, n 52, p. 3-8.

CTGREF, 1977. - Comportement et toxicité à l'égard des poissons de certains fongicides (dithiocarbamates). Incidences sur les piscicultures. Inf. Tech. CTGREF $n^{\circ} 1$.

Cuinat R., 1980..- Le saumon du bassin Loire Allier. Saumons, $\mathrm{n}^{\circ} 34$, p. 26-32.

DODSON J.J., LegGetT W.C., JoNES R.A., 1972. - The behavior of adult American shad (Alosa sapidissima) during migration from salt to fresh water as observed by ultrasonic technics. $J$. Fish Res. Bd. Canada, 29, p. 14451449.

Elie P., Rigaud C., 1984. - Etude de la population d'anguilles de l'estuaire et du bassin versant de la Vilaine: pêche, biologie, écologie. Examen particulier de l'impact du barrage d'Arzal sur la migration anadrome (civelles). Tome I, 175 p., Tomes II et III, 175 p., CEMAGREF - ALA, Bordeaux.

FONTAINE M., 1984. - Influence des pollutions sur les migrations. Saumons, $\mathrm{n}^{\circ} 48$, p. 7-10.

GueneAu P., 1984. - Le Radio-Pistage des saumons en Loire. Bulletin de liaison du Conseil supérieur de la péche, $\mathrm{n}^{\circ} 35$, octobre 1984 , p. 6-8.

LARINIER M., TrAVADE F., 1982. - Les poissons migrateurs aux Etats-Unis. Ouvrages de franchissement des barrages. Programmes de restauration des populations. Rapport EDF. $\mathrm{HE} / 31.82 .56,53 \mathrm{p}$.

LeYNAud G. et TROCherie F., 1980. - Effets toxiques des pollutions sur la faune piscicole, in : Pesson P. La pollution des eaux continentales. Gauthier-Villars Paris, p. 147-168.

NORDENG H., 1977. - A pheromone hypothesis for homeward migration in anadromous salmonids. Oikos, 28, p. 155-159.

Roule L., 1929. - Les cours d'eau de notre pays considérés par rapport à la montée du saumon. Revue des Eaux et Forêts, p. $1-5$.

RUGGLES C.P., 1980. - A review of downstream migration of Atlantic Salmon. Canadian Technical Report of Fisheries and Aquatic Sciences $\mathrm{n}^{\circ} 952.39 \mathrm{p}$.

SAglio P., 1979. - Communication chimique et migration reproductrice chez les salmonidés. Bulletin Français de Pisciculture, $\mathrm{n}^{\circ} 275$, p. $72-82$.

SAGLio Ph., 1983. - Olfaction et homing chez les salmonidés. Saumons, $n^{\circ} 44$, p. $3-4$.

Sprague J.B. et DruRY D.E., 1968. - Avoidance reactions of salmonid fish to representative pollutants. 4 th Intern. Conf. Wat. Poll. Res., Prague 1968.

StaBell O.B., 1984. - Rôle joué par l'olfaction et la génétique dans le comportement de "homing " chez le saumon atlantique. Saumons, $\mathrm{n}^{\circ} 51$, p. 21-25. 


\section{Le Président remercie M. Leynaud pour son exposé.}

M. TRIVELLATO intervient pour souligner l'influence des ouvrages hydrauliques, pas toujours négative, sur les hydrogrammes des crues, et par conséquent sur la migration mais également sur les modifications de la morphologie fluviale, modification apportée au charriage des matériaux et au cheminement des sédiments (accumulation en amont et régression en aval) avec effets sur les frayères.

Il pose le problème de la recherche de solutions pour les exploitants de barrages, et pense qu'il y aurait des études intéressantes à faire sur la modification du lit des rivières.

M. LEYNAUD précise que l'objectif de l'exposé était de décrire des obstacles mais pas les solutions qui demanderaient des développements plus longs. Mais il est d'accord avec M. TRIVEL LATO quant aux effets indirects des barrages avec les modifications de l'habitat, y compris les modifications géométriques. Par exemple le creusement des lits par des extractions de matériaux, le changement du régime hydrologique ou l'existence de retenue en amont, entraînent un transport des sédiments avec des zones de dépôts nouvelles. Ceci, lié parfois à des variations de hauteurs d'eau, constitue des modifications auxquelles sont sensibles les poissons. Les relations existant entre les caractéristiques hydrodynamiques et les peuplements piscicoles constituent un problème important et très complexe sur lequel des organismes scientifiques devraient mettre en commun leurs compétences.

$M$. BAILLYY s'interroge sur le débit réservé pour des microcentrales, prévu par la loi Pêche ( $10 \%$ du module interrannuel). I demande si ce seuil de $10 \%$ est compatible avec la réalité d'une vie piscicole normale, dans le cas contraire quelle est la valeur qu'il faudrait prendre en compte $(20 \%$ lui semblerait un chiffre plus cohérent).

M. LEYNAUD précise que la question des débits réservés (débits minimaux) est un problème mal connu ou du moins mal quantifié. La loi Pêche a prévu un débit minimum chiffré unique qui, dans certains cas, peut se révéler insuffisant. Il faut noter que le critère déterminant retenu par la loi (article 410) n'est pas ce chiffre, il est d'ordre biologique (garantie permanente de la vie, de la circulation et de la reproduction des espèces autochtones); il peut être mis en œuvre si nécessaire.

$\mathrm{Si}$ des moyens adéquats existaient pour effectuer des études fines et détaillées, cette notion de débit réservé pourrait être abordée de façon plus rationnelle au cas par cas pour la détermination chiffrée de débits " acceptables".

Il regrette que faute de moyens, cette démarche ne soit pas réalisable pour la majorité des demandes.

Dans cette sorte de course entre les aménageurs et les gestionnaires de l'eau, ces derniers ont toujours un peu de retard, ce qui conduit à la fixation de seuils de portée générale représentant le plus souvent des contraintes minimales.

Ce problème se retrouve d'ailleurs au niveau des objectifs de qualité et des normes de rejets.

M. TOULLIC aborde le problème de l'eutrophisation des cours d'eau et voudrait savoir si le développement massif de certaines algues planctoniques peut tuer le poisson.

M. LEYNAUD indique que le développement dalgues peut induire la mort du poisson de deux façons différentes.

- dans certains cas extrêmes par la secrétion de substances toxiques; on a pu constater effectivement de tels accidents; - par la modification des caractéristiques de la teneur en $\mathrm{O}_{2}$ de l'eau: fortes sursaturations en $\mathrm{O}_{2}$ dans la journée suivies de dépressions, la nuit, toutes aussi marquées. La sursaturation en $\mathrm{O}_{2}$ ne présente pas, en elle-même, de danger, c'est la variation importante de ce paramètre qui soumet les organismes à des stress importants. On peut arriver en période nocturne à l'anoxie, donc à des mortalités brutales rapides, difficilement identifiables parce que le matin le taux d'oxygène est tout à fait normal. Il est important de suivre le cycle complet de $\mathrm{I}^{\prime} \mathrm{O}_{2}$ pendant $24 \mathrm{~h}$ au moins.

Le développement important des algues entraîne également une élévation du $\mathrm{pH}$ qui, si elle est associée à la présence d'azote ammoniacal, entraîne des problèmes importants de toxicité pour le poisson.

On peut donc avoir une superposition d'actions qui, malheureusement, vont toutes à l'encontre du poisson.

M. BUTTIKER s'interroge sur le cumul des obstacles qui peut engendrer la diminution du stock des géniteurs et donc compromettre, dans certains cas, la reproduction. Il demande si l'on peut quantifier la réduction du nombre de géniteurs et dire pour les différentes espèces à partir de quel nombre d'obstacles on peut voir un nombre suffisant de géniteurs parvenir aux frayères.

Pour M. LEYNAUD la question est difficile car liée à l'efficacité des ouvrages de franchissement. Si les obstacles sont mal aménagés, la limite est très rapidement atteinte au bout du $3^{e}$ ou $4^{e}$ obstacle, parfois même, dans certains cas, au bout du premier. Aucun dispositif ne présente une efficacité de $100 \%$ (tout au moins sans retard pour la migration).

Les biologistes peuvent dire à partir de quel moment on n'a plus assez de géniteurs pour utiliser les potentialités du milieu qui dépendent de la capacité d'accueil et de l'importance du réseau hydrographique. Mais on n'a pas encore pu quantifier réellement l'effet de barrages successifs correctement équipés de passes à poissons.

Cette question est très importante car le nombre de barrages successifs, même correctement aménagés, ne peut être inconsidérément augmenté sans compromettre à un certain niveau les peuplements de migrateurs.

La quantification exacte est un problème délicat. Actuellement, en France tout au moins, on commence à construire des ouvrages de franchissement qui ont une bonne efficacité même si elle n'atteint pas $100 \%$. Pour le franchissement intervient non seulement l'ouvrage lui-même mais aussi sa situation, le débit, la facilité avec laquelle les poissons migrateurs vont trouver le passage qu'on leur propose. Le même problème se pose à la descente : les passages soit par les déversoirs, soit par les turbines, constituent des dangers qui peuvent réduire dangereusement les effectifs de juvéniles puisque les pertes mesurées au cours de l'avalaison peuvent atteindre jusqu'à $95 \%$ sur certaines rivières équipées.

M. PORCHER désire compléter la réponse de M. LeYNAUD sur le nombre d'obstacles. Selon lui il n'y a pas de réponse générale mais une réponse particulière au cas considéré.

Un barrage a plusieurs effets : empêcher les migrateurs de passer, ennoyer une partie du cours d'eau amont donc supprimer des frayères, modifier des zones de production de juvéniles. Il faut donc étudier, sur le site même, dans les conditions sur lesquelles on travaille, quelles sont les conséquences que l'on peut attendre de cet ouvrage, et, à l'aide d'un exemple chiffré, voir quelle est la surface de production utile en juvéniles. Les barrages installés sur le bassin de la Loire ont diminué de $90 \%$ environ la capacité initiale de production en saumon : il y a toujours du saumon dans ce bassin, mais le stock est réduit en conséquence.

A partir du moment où l'on modifie le milieu naturel, on modifie aussi les populations de poissons et le choix à faire est un choix politique, à savoir jusqu'où peut-on aller dans la modification de l'environnement. 